\title{
A WebGIS platform for mobile robot navigation developed with free and open source software
}

\author{
Michele Mangiameli*, Giovanni Muscato, Giuseppe Mussumeci
}

\begin{abstract}
Nowadays more and more applications require a mobile robot able to operate autonomously in an outdoor environment. The accuracy and precision of mapping systems for the working environment is particularly important for the management, control and efficiency of the activity of the mobile robot. For these reasons, mapping systems must be consistent and efficient, and capable to represent in real-time the surroundings, thus allowing the robot, equipped with a system of autonomous movement, the exploration of unknown environments. Here we present a new Web GIS platform able to track the navigation of mobile robots on a georeferenced cartographic data and to assign possible paths or waypoints. The WebGIS platform has been developed using only free and open source software and has been customized with useful tools for the management of the navigation of mobile robots.
\end{abstract}

Keywords - WebGIS, Spatial Database, Robotics

\section{Introduction}

The use of autonomous robots is a valuable tool for the exploration of hostile environments and sites affected by natural disasters (Figure 1), i.e. unstable areas or structures, underground cavities, polluted sites, buildings at risk of collapse, volcanic phenomena $[1,2,3]$. The navigation of a robot is usually monitored and controlled by inertial platforms and in outdoor applications by means of satellite positioning, as the GPS technology [4].

Recently it has been shown how the correct georeferencing and interpretation of assigned routes and trajectories covered by a mobile robot, can be managed by GIS (Geographic Information System) technologies [5, 6, 7]. The GIS environment allows managing the navigation of mobile robots on georeferenced map and acquiring any additional information from the sensors installed on the robot using a supplementary spatial database. However, the cartography often lacks in precision, being based on supports not accurately georeferenced, i.e. Google Maps [8]. In other cases, the systems developed are based on proprietary software, thus not easily customizable [9] or show very limited functionalities [10]. In this paper we presents an innovative Web GIS platform developed with free and open source technologies for the management of mobile robot navigation. The GIS architecture proposed allows the real-time monitoring of the robot position on a georeferenced map using the information provided by the GPS receiver installed on board of the robot, assigning possible paths or way points and scanning possible obstacles for the navigation.

Michele Mangiameli, Giovanni Muscato, Giuseppe Mussumeci

University of Catania, Italy
This architecture has been enriched using a direct link with a spatial database to guarantee a unique software tool for the management of the spatial data, allowing the exploitation of all instruments provided by the spatial database and the characteristics available within the DBMS (DataBase Management System) [11, 12]. With such an architecture, different kinds of access policies can be integrated.

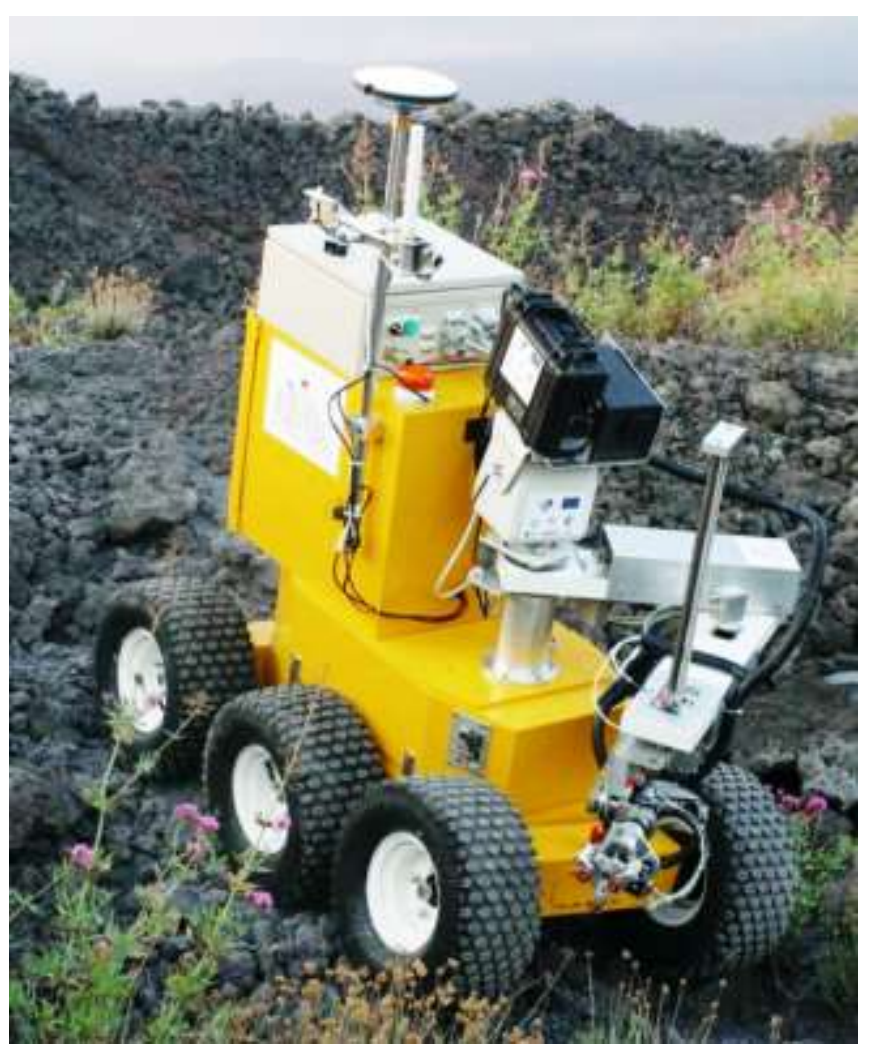

Figure 1. ROBOVOLC, a mobile robot for volcano exploration (http://www.robovolc.diees.unict.it/)

\section{The system architecture}

The architecture of our system has been developed with the purpose to real-time track the robot position in the GIS environment using the information collected through the GPS. In particular, the architecture shows a flexible modular structure where the GPS communicates with both the database and the WebGIS platform (Figure 2). This platform allows the distribution of geo-spatial data in internet and intranet networks, exploiting the analysis derived from GIS software and by means of classical features of web-based publish geographical information on the World Wide Web [11, 13]. 
Proc. of the Second Intl. Conf. on Advances in Information Processing and Communication Technology - IPCT 2015

Copyright (C) Institute of Research Engineers and Doctors, USA .All rights reserved.

ISBN: 978-1-63248-044-6 doi: 10.15224/ 978-1-63248-044-6-19

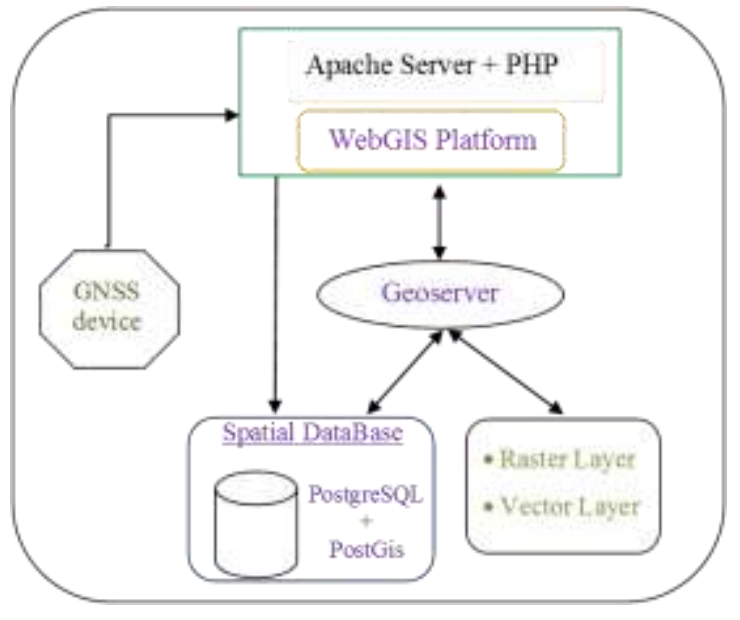

Figure 2. General architecture beyond our Web GIS platform

Like a classical web architecture, a WebGIS system is based on standard client-server functionality. The GPS device is installed on board of the robot and sends information about its location using the standard communications GPS data protocol NMEA. Using a PHP code, located within the Apache server, the parameters are set to communicate with the GPS. In this way, information related to the position of the robot can be used by the Web GIS platform for the tracking of the robot on a georeferenced cartography.

But as the NMEA string is composed by different expressions, it is needed to select the sentence that contains the most complete information. In particular, the most complete NMEA string is identified with the prefix "\$ GPRMC" (Recommended Minimum GPS Data), with all information separated by commas, as follows:

\section{\$GPRMC,231712,A,3731.2318,N,01506.2156,E,1.3,333.7,210313,2.0,E,D*10}

The first value is the identifying prefix of the NMEA string, the second value is the time in UTC (Coordinated Universal Time) format, the third value represents the status of the GPS device, which can be A=active or V=Void, the fourth and fifth values are respectively the Nord latitude and East longitude, the eighth value represents the speed expressed in knots, the ninth value shows the direction, the tenth value shows the date of acquisition, the eleventh and twelfth values indicate the parameters of magnetic variation, and the last value is a checksum.

To use this information from the GPS inside the Web GIS platform, it was necessary to structure the source code by selecting only this string from NMEA complete listing and choosing only the strings that identify an active GPS state.

Finally, the latitude and longitude of the selected NMEA string are automatically converted within the source code of the Web GIS platform from degrees, minutes and seconds to sexagesimal units for a correct georeferencing on the raster cartography map with a WGS84 datum (Figure 3).

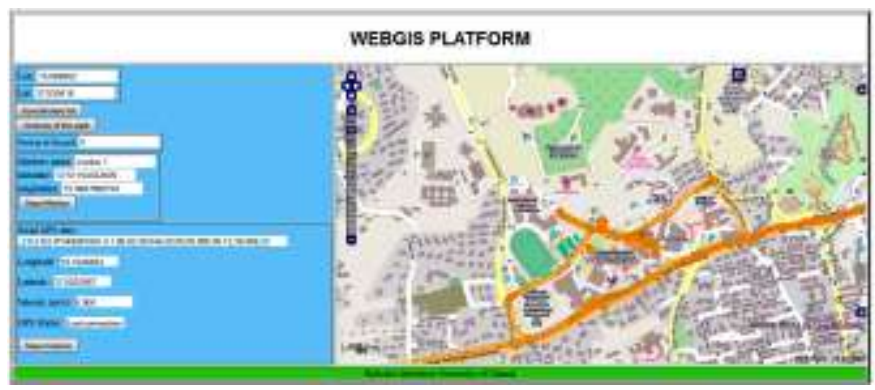

Figure 3. Robot track on the OpenStreetMap cartographic support with a WGS84 datum

The information coming from the GPS can be directly inserted inside the spatial database using another PHP code. In this case, specially developed parameters are set of communication with the GPS device, after the NMEA string with prefix GPRMC is being selected.

Since the latitude and longitude, as well as other information within the NMEA string are alphanumeric data without spatial characteristic, a software procedure has been developed to convert the information relative to the position of the robot, from purely numerical parameters to geographical coordinates manageable in a GIS environment as vector themes.

The procedure involves the use of code developed in PHP language and spatial queries built using SQL language, for taking information from the NMEA string, attach them to a datum and insert into the spatial DB managed by the Database Management System (DBMS) PostgreSQL with PostGIS spatial extension to manage information with geographical characteristics (Figure 4).

To explain in detail the software process, two phases of operation can be distinguished.

In the first step, a table space within the PostgreSQL DBMS is created that hosts the coordinates relative to the robot position together with all useful information extracted from the NMEA string.

In the second phase, the NMEA strings with the prefix \$GPRMC are detected, the columns for latitude and longitude, and using spatial queries executed inside the PHP code, the topographic datum is associated and subsequently are automatically included in columns latitude and longitude of the spatial table created (Figure 5).

The spatial database is PostgreSQL with PostGIS as spatial extension, and is developed with free and open source software. Using the same procedure, other information can be extracted from the NMEA sentence, e.g. the time of acquisition and the altitude. To expedite the process, the first phase of the creation of the spatial table in PostgreSQL can be performed directly inside the PHP code by inserting the query space for the creation of geometrical table. The spatial table containing the data position of the robot is connected to the web platform using GeoServer. 
Proc. of the Second Intl. Conf. on Advances in Information Processing and Communication Technology - IPCT 2015

Copyright $\odot$ Institute of Research Engineers and Doctors, USA .All rights reserved.

ISBN: 978-1-63248-044-6 doi: 10.15224/ 978-1-63248-044-6-19

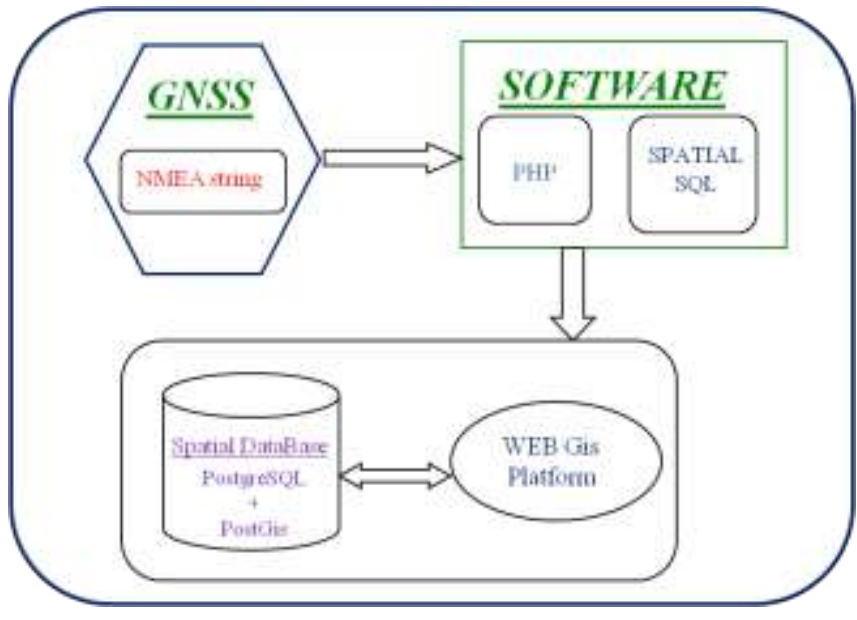

Figure 4. Software architecture to manage the position of the mobile robot

GeoServer is an open source software server written in Java that allows sharing and editing geospatial data(http://geoserver.org/display/GEOS/Welcome). Designed for interoperability, it publishes data from any major spatial data source using open standards. With this technique, the tracking of the robot is managed within the web platform as a Web Feature Service (WFS) layer setting a refresh in javascript function of the platform OpenLayer where the parameters of the WFS layer are set. With this technique different raster cartographic supports or vector layers can be queried and managed for the visualization of possible obstacles for the robot navigation.

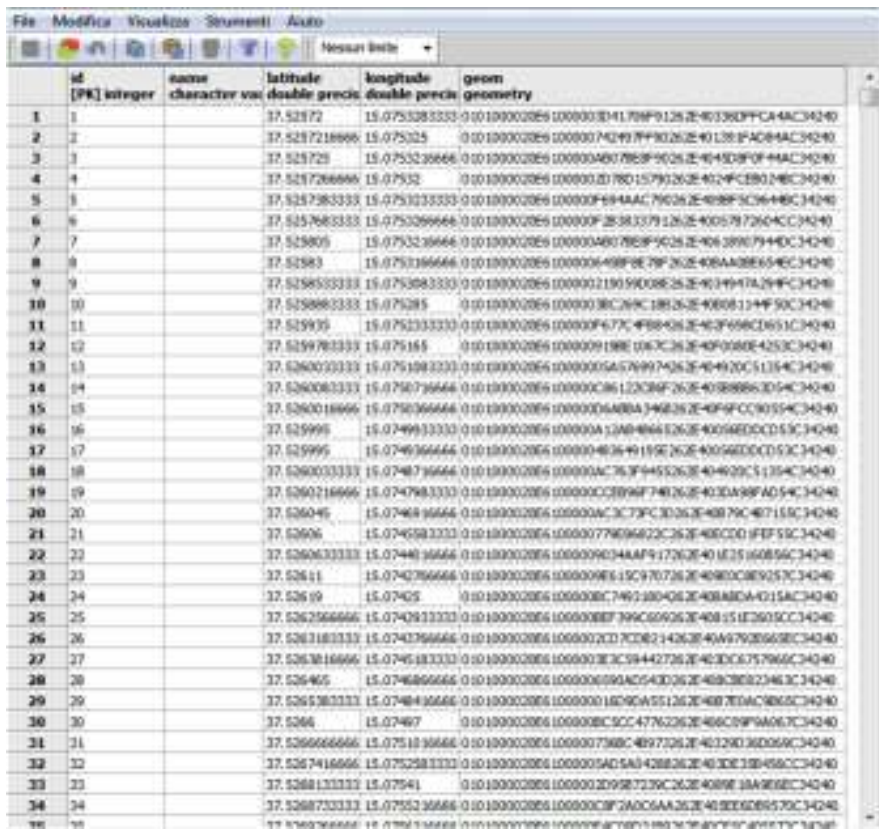

Figure 5. The spatial table containing the positional data of the robot
The table geometry can be thus used as vector layer in a GIS Desktop or other Web GIS platforms to show the position of the robot on a georeferenced cartographic supports, orthophotos, etc., with the WGS84 datum. As desktop GIS platform, we used the free e open source QGIS software, where the geographical database is connected with the platform through the tool "Add PostGIS layer".

The advantages of the proposed architecture for the management of the mobile robot navigation are multiple: (i) the software technology is free and open source, thus easily downloadable from the web at no costs; (ii) the architecture can be used with any GPS device since the NMEA standard protocol is used as output; (iii) the tracking of the robot is managed on raster supports georeferenced with the classical topographic approaches and tools available in real time in the desktop and WebGIS platforms because the waypoints are dynamically stored in the database . In particular, the WebGIS platform has been totally customized using JavaScript libraries of OpenLayers.

\section{The Web GIS platform}

The Web GIS platform able to manage the navigation of mobile robots has been developed and customized with the free JavaScript library OpenLayers [http://openlayers.org/]. This Web GIS platform is characterized by a large map area for displaying the active layers; a retractable layer tree on the right, where all available layers are listed; a retractable reference map at the bottom right, where the map area is represented with respect to a global map; and a toolbar at in the top right corner of the map (Figure 6).

The toolbar contains standard icons with useful tools for navigating the map, i.e. a zoom, pan and standard icons associated with functionalities specifically developed to manage the navigation of mobile robots. Using the standard libraries, simple geometric primitives can be digitized on the cartographic support, even if they are simple drawings, not usable as georeferenced spatial data. To overcome this limitation, a JavaScript and PHP application has been implemented to digitize geometric objects available in the Web GIS platform and manageable as vector layers. This part of the code developed represents an easy way to create the georeferenced vector themes in a WebGIS environment. Using this procedure, they can be characterized by spatial information, i.e. the latitude and longitude of each vertex, which can be saved as waypoints in text file used by the navigation system of the robot. With this procedure, the Web GIS platform can be used to assign the path to the robot. By clicking on the toolbar, polylines, paths or individual points can be also digitized. Other functionalities to edit these geometries, i.e. the "delete feature" tool, where the feature is automatically selected and removed, and the "save waypoints" tool for saving them in a text file have been also implemented. The vector themes created can be turned on or off using the layer tree (Figure 7). 


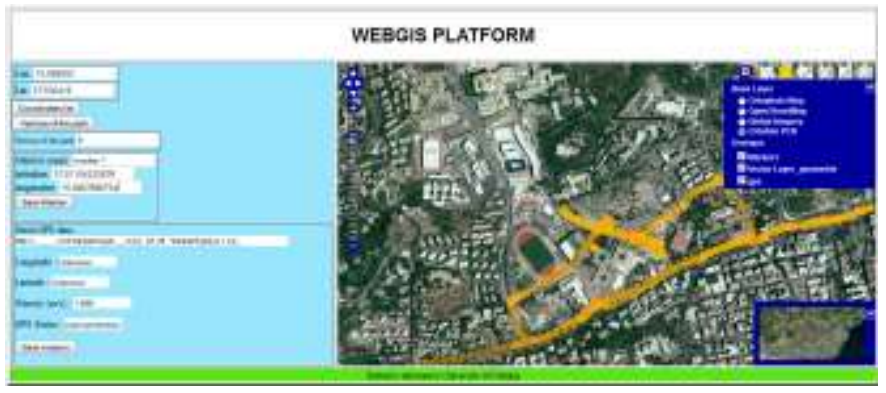

Figure 6. Interface of the Web GIS platform

Moreover two bars have been inserted on the left and right bottom corners for scaling and for dynamically representing the coordinates when the mouse moves on the map.

On the left side of the Web GIS platform, the coordinates of the digitized paths and the total number of vertices can be displayed. These latter ones are visible by either clicking on a button or in real-time while digitizing a route or waypoints.

A mask for a dynamic data entry of a spatial database has been also included. In particular, by entering the information in the name field, and the coordinates in latitude and longitude fields, the information can be inserted in the spatial database and automatically georeferenced. The information is displayed on the cartography support as a layer with punctual geometry.

A mask control for the GPS receiver has been inserted, where the NMEA string, longitude, latitude, speed and connection status that can be are displayed. The connection status may be valid, unknown or lost.

We tested the Web GIS platform developed for managing the navigation within the university campus of the Faculty of Engineering of the University of Catania. As cartography map, we used a orthophotos with a WGS84 datum, taken as Web Map Service (WMS) service from the National Cartographic Portal (http://www.pcn.minambiente.it/GN/). The position of the robot has been managed within the Web GIS platform using the NMEA string in output from the GPS installed on board of the robot. Using the tools within the Web GIS platform, possible waypoints were assigned to the mobile robot, whose position was tracked and monitored in real time. With this approach, the position of the robot was perfectly georeferenced thanks to the high accuracy of the cartographic support and GNSS correction system used. The data related to the position of robot were inserted within the spatial database in real time and information from the sensors mounted on board of the robot (e.g. temperature sensors and gas) were associated to each pair of coordinates (latitude and longitude). In this way, any data or environmental parameter detected by the sensors is perfectly georeferenced and managed within the GIS environment.

Finally, using a specific button available within the platform, the mission of the robot can be saved in a csv file (named using the date and hour of the mission), which reports the values contained in the table of the spatial database.

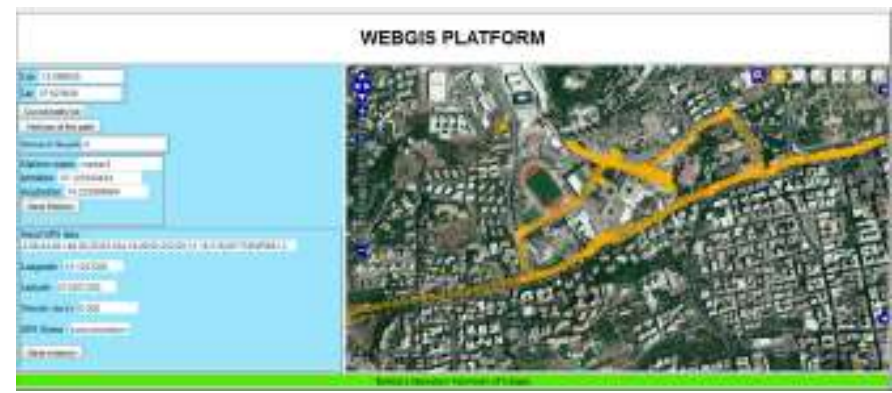

Figure 7. Toolbar for digitizing possible obstacles identified on the cartography support

\section{Discussion and conclusions}

The software architecture described in the previous paragraphs and successfully tested, provides a valuable tool for the tracking of mobile robots (UAV and UGV). The software architecture is expandable with additional cartographic raster support and usable with any GPS device, thanks to the customization of the source code that uses data the NMEA standard protocol as GPS output for the representation of the position of the robot on a georeferenced raster support.

The tests performed on the platform have confirmed the good functionality and precision of the tool, developed and customized, to assign paths and waypoints for the robot.

In conclusion, the software architecture and Web Gis platform developed and customized, result to be a good tool for the management of mobile robots in areas inaccessible or hazardous for humans. Possible scenarios of application include such areas subject to disasters, such as floods or volcanic eruptions, where the intervention of autonomous robots managed through the proposed architecture allows exploring areas inaccessible or dangerous for rescue teams and collecting useful information directly transmitted to the control station.

\section{References}

[1] R. Robin Murphy, S. Tadokoro, D. Nardi , A. Jacoff MS, P. Fiorini , H. Choset, A. M. Erkmen Search and Rescue Robotics, Springer Handbook of Robotics, 2008, p.p.1151-1173.

[2] M. Maugeri, E. Motta, G. Mussumeci, E. Raciti, "Lifelines seismic hazard: a GIS evaluation". In Seventh International Conference on Earthquake Resistant Engineering Structures (ERES), Cyprus, 2009, doi: 10.2495/ERES090351.

[3] S.Tadokoro, Rescue robotics. Berlin: Springer,2010.

[4] F.Bonaccorso, L. Cantelli, D. Longo, D. Melita, G. Muscato, M. Prestifilippo, The U-Go Robot, "A multifunction rough terrain outdoor tracked vehicle for R\&D on autonomous navigation algorithms", Proceedings of the 5th IARP Workshop on Robots for Risky Interventions and Environmental Surveillance Maintenance, Leuven, 2022 June 2011.

[5] AA. Alesheikh, A.S. Niaraki, M. Varshosaz, H. Helali, "Satellite Location \& Navigation of Vehicles on the International Transport Corridor of Iran, Russia and India”, Proceedings of Geomatics (ISPRS) 2002. 
Proc. of the Second Intl. Conf. on Advances in Information Processing and Communication Technology - IPCT 2015

Copyright $(\odot$ Institute of Research Engineers and Doctors, USA .All rights reserved.

ISBN: 978-1-63248-044-6 doi: 10.15224/ 978-1-63248-044-6-19

[6] K. Do-Hyun, K. Min-Soo, "Web GIS Service Component Based On Open Environment", Geoscience and Remote Sensing Symposium,

vol.6, p.p.3346-3348,IEEE International DOI: 10.1109/IGARSS.2002.1027178.

[7] M. Mangiameli, G. Muscato, G. Mussumeci, "Development of software procedures for assigning optimal paths to autonomous robots in open source GIS environment", in Proceedings of MIMOS 2012, Rome , 9-11 ottobre 2012.

[8] L. Yu-Cheol , Christiand , Y. Wonpil, and K. Sung-Hoon "Topological Map Building for Mobile Robots Based on GIS in Urban Environments", 8th International Conference on Ubiquitous Robots and Ambient Intelligence 2011 in Songdo ConventiA, Incheon, Korea.

[9] M. Ceccarelli, F. Cioffi, M. Di Capua, "Dynamic webgis and tracking of mobile devices", Proceedings (FOSS4G Free And Open Source Software for Geoinformatics - Losanna - Settembre 2006).

[10] Y. Daisuke, S. Xianfeng, R. Venkatesh, "Development of track log and point of interest management system using Free and Open Source Software" in Appl Geomat 2010,vol. 2, p.p. 123-135 DOI 10.1007/s12518-010-0028-7

[11] E. Khoumeri, D. Benslimane, "Web GIS For Multiple Representation Data" Digital Information Management, ICDIM '07. 2nd International $\begin{array}{llllll}\text { Conference on } 2007 & \text { vol.1 p.p } & 188-192 & \text { DOI: }\end{array}$ 10.1109/ICDIM.2007.4444221.

[12] X. Daoxun, X. Xiaoyao,"Web GIS Server Solutions using Open-Source Software", Open-source Software for Scientific Computation (OSSC), IEEE International Workshop 2009, p.p.135-138 DOI: 10.1109/OSSC.2009.5416738.

[13] D. Famoso, M. Mangiameli, P. Roccaro, G. Mussumeci, F.G.A. Vagliasindi, "Asbetiform fibers in the Biancavilla site of national interest (Sicily, Italy): review of environmental data via GIS platforms" Reviews in Environmental Science and Bio-technology, ISSN: 15691705, DOI 10.1007/s11157-012-9284-9. 